Tohoku J. Exp. Med., 2006, 208, 33-39

\title{
Increased Serum Malondialdehyde Levels in Chronic Stage of Ischemic Stroke
}

\author{
Levent Sinan Bir, Süleyman Demir, ${ }^{1}$ Simin Rota ${ }^{1}$ and Mehmet KöSeoĞLu ${ }^{2}$ \\ Department of Neurology, 'Department of Biochemistry, Pamukkale University \\ Medical School, Denizli, Turkey, and \\ ${ }^{2}$ Department of Biochemistry, Atatürk Education and Research Hospital, İzmir, \\ Turkey
}

BIR, L.S., Demir, S., Rota, S. and KöseoĞLu, M. Increased Serum Malondialdehyde Levels in Chronic Stage of Ischemic Stroke. Tohoku J. Exp. Med., 2006, 208 (1), 33-39

- Serum lipoperoxidation products such as malondialdehyde (MDA) reflect oxidative stress. There are contradictory results addressing the levels of lipoperoxidation products in chronic phase of ischemic stroke. In the present study, we aimed to determine the serum MDA levels in stroke patients 6 months after the cerebrovascular accident. We also compared serum MDA levels in two major groups of patients with ischemic stroke resulting from small vessel and large vessel diseases, respectively. Serum MDA levels of thirtyeight patients who had ischemic stroke (19 with atherothrombotic ischemic stroke and 19 with lacunar infarction) and 30 healthy volunteers were measured. While there was no significant difference in serum MDA levels between the chronic ischemic stroke subgroups $(p=0.795)$, the serum MDA levels of patients with atherothrombotic ischemic stroke $(p<$ $0.001)$ or with lacunar infarction $(p<0.001)$ were significantly higher compared to the control group. We also demonstrated that serum MDA levels of the patients with and those without hypertension ( $p=0.846)$, diabetes mellitus $(p=0.891)$, or dyslipidemia $(p=0.38)$ were not significantly different. In conclusion, serum MDA levels were elevated in chronic stroke patients with small or large vessel diseases. To the best of our knowledge, this is the first report showing that serum MDA levels of these two groups are not sigificantly different. Furthermore, serum MDA levels do not differ solely by the existence or nonexistence of hypertension, diabetes mellitus or hyperlipidemia. —— ischemic stroke; chronic stroke; malondialdehyde; MDA; oxidative stress

(C) 2006 Tohoku University Medical Press

The development of a focal neurological deficit is designated stroke, if the cause of the deficit is thought to be consequence of a local disturbance in the cerebral circulation (Garcia et al. 1992). Stroke is classified in two major forms based on the pathology of underlying focal brain injury into either ischemic infarction or hemorrhage. Ischemic infarction can also be classified into various subgroups based on the mechanism of the ischemia and the type and localization of the vascular lesion (Sacco 2000). Major ischemic stroke subgroups are accepted as infarct with large arterial thrombosis, lacunar infarction, and embolism attributed to cardiac or transcardiac sources (Mohr and Sacco 1992). The former two subgroups possess the same risk factors, especial-

Received September 6, 2005; revision accepted for publication October 14, 2005.

Correspondence: Dr. Simin Rota, PK 259 Yenişehir, 06443 Ankara, Turkey.

e-mail:siminrota@yahoo.co.uk 
ly hypertension (HT) and diabetes mellitus (DM), and both are the results of atherosclerosis (DeGraba et al. 1992). While large arteries are affected in the first type, in the second the arterioles are the targets. The small arterial pathologies underlying the lacunar infarctions are microatheroma, lipohyalinosis, fibrinoid necrosis, CharcotBouchard aneurysm, and microembolism. Since in advanced hypertensive patients, atherosclerotic lesions can be seen even in small arteries of 100 to $400 \mu \mathrm{m}$ diameter, microatheroma is believed to be the most common mechanism underlying lacunar infarction (Mohr 1992).

Inflammation process is an important aspect during the process after cerebral ischemia (Tamam et al. 2005). Free radical production, leading to oxidative stress contributing to brain damage is increased in ischemic stroke. For better understanding of the pathophysiology of stroke and identifying the subgroups of patients that would be receiving therapeutic intervention, measurement of oxidative stress parameters would be extremely important. Some biological substances that might be potential peripheral markers in stroke are being investigated, since the direct measurements of free radicals and oxidized molecules in brain are difficult in humans (Cherubini et al. 2005). Significant increases of lipoperoxidation products or decreases of some antioxidants in plasma are reported in stroke patients and the presence of oxidative stress in stroke has been judged by these indices (Alexandrova and Bochev 2005).

One of the well known lipoperoxidation products is malondialdehyde (MDA) (Alexandrova and Bochev 2005; Cherubini et al. 2005). Lipid peroxidation, leading to the production of unstable conjugated dienic lipid hydroperoxides results from the decomposition to aldehydes as MDA and 4-hydroxynonenal (HNE), dienals and alkanes. Except for dienals and alkenes, other products are the mainly measured products for the detection of oxidative stress (Dotan et al. 2004).

In several studies, higher serum MDA levels in acute stroke patients compared to healthy controls were shown (Sharpe et al. 1994; Re et al.
1997; Belch et al. 1998; Iavorskaia et al. 2000; Demirkaya et al. 2001; Polidori et al. 2002; Bolokadze et al. 2004). To our knowledge there are no data concerning the serum MDA levels in these individuals before they have cerebrovascular accident. Since there is a close relation between the lipoperoxidation products and HT, $\mathrm{DM}$, dyslipidemia, or atherosclerosis, that are frequently present in stroke patients, high serum MDA levels detected in these individuals might be regardless of stroke. Related studies comparing serum MDA levels of acute stroke patients with healthy population were performed in acute phase of stroke, immediately after the accident. However, there are only a few studies addressing the lipoperoxidation products in chronic stage of stroke with conflicting results (Wehr et al. 2000; Alexandrova et al. 2003). For this contradiction, in this study we planned to determine the serum MDA levels of chronic stroke patients (6 months after the cerebrovascular accident). We also aimed to compare serum MDA levels of two major subgroups of ischemic stroke patients resulting from small vessel and large vessel diseases, respectively. We also compared serum MDA levels in chronic stroke patients with and those without HT, DM, or dyslipidemia.

\section{Materials and Methods}

\section{Patients}

Patients included in this study were chosen among the stroke patients who had been followed up in our neurology clinic. For this study these patients were invited to the neurology outpatient clinic six months after the cerebrovascular accident. Thirty eight patients (20 men and 18 women) who had ischemic stroke (19 with atherothrombotic large artery disease and 19 with small artery disease with lacunar infarction) and 30 healthy age and sex matched (16 men, 14 women) volunteers as the control group were enrolled in this study. The patients with small artery disease had lacunar syndrome. The routine biochemical analysis of the patients had been performed. According to our stroke research protocol all stroke patients had underwent to carotid and vertebrobasilar doppler ultrasonographic and cardiological examination, including electrocardiography and echocardiography. Also serum markers of vasculitis (antinuclear antibody, anti-DNA, antinuclear anticytoplasmic anti- 
body, anticardiolipin antibody, Anti-Ro, Anti-La) had been measured and they were all in normal limits. Hematological parameters were also in their normal limits. Patients with stroke related to cardiac or transcardiac embolism, vasculitis, hematological abnormalities were not included to the study. For each patient medical history of HT, DM, dyslipidemia were recorded. A total of 38 patients in atherothrombotic and lacunar groups were evaluated according to the presence of HT, DM and dyslipidemia. In atherothrombotic and lacunar groups consisting of 38 patients, 23 patients $(60.53 \%)$ had HT, 9 (23.69\%) DM and 24 (63.16\%) dyslipidemia. All the patients were under medication of $100 \mathrm{mg} /$ day acetylsalycilic acid. Some of the individuals in the patient group were also using antihypertensive drugs, and/or insulin and/or statins. The stroke patients with co-existing coronary artery disease, peripheral vascular disease were not included to the study. In control group, individuals with a history of stroke, coronary artery disease, peripheral vascular disease and other systematic diseases were also not included to the study. None of the subjects in the control group were taking any systemic medications. All the individuals included to the study were nonsmokers. Cranial Magnetic Resonance Imaging (MRI) was performed in each patient. In atherothrombotic group large infarct area in the middle cerebral artery tertiary and in lacunar group multiple lacunes and ischemic gliotic areas were detected. In atherothrombotic group, patients with coexistent lacunar infarcts detected by MRI and in lacunar group patients with coexistent atherosclerotic findings in carotid and/or vertebro-basilar arteries detected by doppler ultrasonography were not included to the study. In every individual of the control group cranial MRI was also performed to exclude clinically silent cerebrovascular disease. Informed consent was obtained from all of the subjects. All experiments were performed in compliance with the relevant laws.

\section{Method}

For the measurement of MDA, venous blood samples were drawn from the participants after fasting for 12 hours. Samples were collected in serum separator tubes, allowed to clot for $30 \mathrm{~min}$, centrifuged for $15 \mathrm{~min}$ at 2,000 $\times \mathrm{g}$ at room temperature and stored at $-70^{\circ} \mathrm{C}$ until assayed.

Plasma MDA levels were determined by the procedure of Ohkawa et al. (1979). All the chemicals were supplied from Sigma-Aldrich (St. Louis, MO, USA). One $\mathrm{ml}$ of homogenate, $1.5 \mathrm{ml}$ thiobarbituric acid, $1.5 \mathrm{ml}$ acetic acid (pH 3.5) and $0.2 \mathrm{ml}$ sodium dodecyl sulphate were mixed. A set of malondialdehyde standards was also freshly prepared. After mixing, all samples and standards were heated at $95^{\circ} \mathrm{C}$ for one hour. The samples and standards were cooled on ice and MDA was extracted by adding $5 \mathrm{ml}$ 1-butanol: pyridine $(15: 1)$ to each tube. The tubes were centrifuged at 2,000 rpm for $10 \mathrm{~min}$ and the aqueous and organic phases were separated. The absorbance of 1-butanol phase was recorded at $532 \mathrm{~nm}$ and the MDA levels of the samples were calculated by using the absorbance of the standard. The results were expressed as $\mathrm{nmol} / \mathrm{ml}$. The intraassay and interassay coefficient of variations of the MDA assay were $4.2 \%$ and $9.1 \%$ respectively.

\section{Statistics}

Statistical analysis was performed by using by Statistical Package for Social Sciences (SPSS 11.0) for Microsoft Windows. The distribution of sex, frequency of HT, DM and dyslipidemia in the groups were compared by Chi-square tests. The mean age, sex distribution, serum MDA levels of the groups were compared by Kruskal-Wallis test. The subgroups were compared to each other by Mann-Whitney's U-test. The results were expressed as median (minimum - maximum).

\section{Results}

The median ages of the atherothrombotic, lacunar and control groups were 69 (38 - 79), 58 (30 - 79), and 59 (40 - 69) years, respectively. There were no significant differences in the mean age (Kruskal-Wallis test, chi-square $=5.449, p=$ 0.066 ) and sexual distribution (Pearson chi square test, $p=0.473$ ) among the groups. There were no significant differences in the frequency of HT, DM, dyslipidemia among atherothrombotic and lacunar groups (Fischer's Exact test, $p=1.84, p=$ $1, p=0.405$, respectively). Median serum MDA levels were detected as $3.50(1.56-6.82) \mathrm{nmol} / \mathrm{ml}$ in atherothrombotic group, 3.83 (1.17 - 5.66) $\mathrm{nmol} / \mathrm{ml}$ in lacunar group, and $1.69(0.45-3.50)$ $\mathrm{nmol} / \mathrm{ml}$ in control group. The serum MDA levels of each individual were given in Fig. 1. Among the groups there was a significant difference in the serum MDA levels (Kruskal-Wallis test, chisquare $=31.62, p<0.001)$. There was no significant difference in serum MDA levels between atherothrombotic and lacunar groups (Mann- 


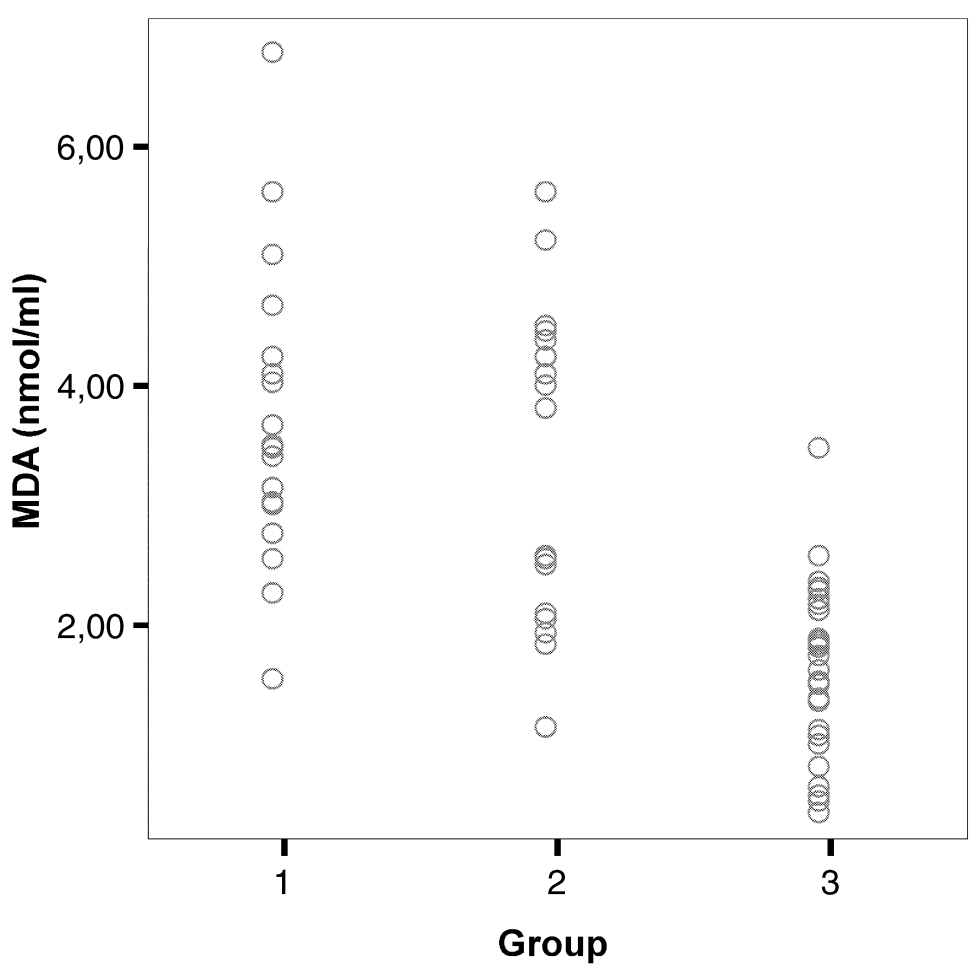

Fig. 1. The distribution of serum MDA levels in the study groups.

Group 1; patients who had atherothrombotic infarction $(n=19)$, Group 2; patients who had lacunar infarction $(n=19)$, Group 3; healthy control $(n=30)$.

TABLE 1. The demographic and biochemical results of the study groups

\begin{tabular}{lcccc}
\hline & $\begin{array}{c}\text { Atherothrombotic } \\
(n=19)\end{array}$ & $\begin{array}{c}\text { Lacunar } \\
(n=19)\end{array}$ & $\begin{array}{c}\text { Control } \\
(n=30)\end{array}$ & $p$ \\
\hline Age (years) & $69(38-79)$ & $58(30-79)$ & $59(40-69)$ & $0.066^{\mathrm{a}}$ \\
MDA (nmol/ml) & & & & \\
Median (min - max $)$ & $3.50(1.56-6.82)$ & $3.83(1.17-5.66)$ & $1.69(0.45-3.50)$ & $<0.001^{\mathrm{a}}$ \\
Mean \pm S.D. & $3.63 \pm 1.33$ & $3.46 \pm 1.38$ & $1.66 \pm 0.69$ & $0.184^{\mathrm{b}}$ \\
Hypertension $^{* *}$ & $9(47.37)$ & $14(73.68)$ & - & $1.00^{\mathrm{b}}$ \\
Diabetes Mellitus $^{* *}$ & $5(26.31)$ & $4(21.05)$ & - & $0.405^{\mathrm{b}}$ \\
Dyslipidemia $^{* *}$ & $5(26.31)$ & $2(10.53)$ & - & - \\
\hline
\end{tabular}

${ }^{*}$ Median (min - max) ${ }^{* *}$ number of subjects $(\%)$

${ }^{a}$ Between all groups (Kruskall-Wallis).

${ }^{\mathrm{b}}$ Between atherothrombotic and lacunar groups (Fischer's Exact test).

Whitney's U-Test, $p=0.795$ ), whereas significant differences were detected between atherothrombotic and control groups (Mann-Whitney's U-Test, $p<0.001)$ and between lacunar and control groups (Mann-Whitney's U-Test, $p<0.001$ ).

The median serum MDA levels of the 23 stroke patients with HT and 15 stroke patients without HT were $3.83(2.8-5.66)$ and 3.86 (2.59 - 5.66) $\mathrm{nmol} / \mathrm{ml}$, respectively. When serum MDA levels of the patients with or without HT were compared, we did not detect any significant difference (Mann-Whitney's U-Test, $p=0.846$ ).

The median serum MDA levels in the 9 stroke patients with DM and 29 stroke patients 
without DM were 3.50 (2.58 - 5.66) and 4.33 (2.08 - 5.66) $\mathrm{nmol} / \mathrm{ml}$, respectively. When serum MDA levels of the patients with and those without DM were compared, we did not detect any significant difference (Mann-Whitney's U-Test, $p=$ 0.891).

The median serum MDA levels in the 24 stroke patients with dyslipidemia and 14 stroke patients without dyslipidemia were 3.83 (2.29 $5.66)$ and $3.86(2.08-5.66) \mathrm{nmol} / \mathrm{ml}$, respectively. When serum MDA levels of the patients with and those without dyslipidemia were compared, we did not detect any significant difference (Mann-Whitney's U-Test, $p=0.389$ ).

\section{Discussion}

In this study, high serum MDA levels were detected in patients who had stroke, after a long period of 6 months. Although there are several studies demonstrating high serum MDA levels in acute phase of stroke (Sharpe et al. 1994; Re et al. 1997; Belch et al. 1998; Iavorskaia et al. 2000; Demirkaya et al. 2001; Polidori et al. 2002; Bolokadze et al. 2004), there are few studies addressing the level of lipoperoxidation products in the chronic stage of the stroke (Alexandrova and Bochev 2005). Alexandrova et al. (2003) demonstrated persistent oxidative damage marker in blood of individuals in the convalescent stage of ischemic and hemorrhagic stroke. They observed a spontaneous hyperactivity of peripheral leucocytes in chronic stroke patients during the 2 nd and 9th month after a severe stroke onset. In another study, no difference was detected in the level of oxidation products in the patients with stroke and the controls. A significant increase was reported only in the group of lacunar strokes (Wehr et al. 2000). Our results are in accordance with the study of Alexandrova et al. (2003) and we agree with the interpretations of the authors stating that the observed contradiction might be the result of stroke heterogeneity, risk factors and methodology used in the studies. Also, differently from Wehl et al. (2000) we determined high serum MDA levels in both groups of chronic stroke patients (either with large artery disease or small artery disease). It can be explained by the pres- ence of similar risk factors especially HT and DM in both ischemic subgroups. Also, the most important mechanism in the development of lacunar infarct is microatheroma as atherosclerotic lesions can be seen even in very small arteries in patients with advanced HT (Mohr 1992). While prior determination of infarct subtype was chiefly based on clinical grounds with heavy reliance on clinical syndrome, neurological examination, and coexisting risk factors, accuracy in the classification of ischemic stroke was achieved by the development of advanced technologies (Mohr and Sacco 1992). For the determination of the participants of the subgroups, we used MRI and carotid and vertebro-basilar arteries doppler ultrasonography. In atherothrombotic group, patients with coexistent lacunar infarcts detected by MRI and in lacunar group, patients with coexistent atherosclerotic findings in carotid and/or vertebro-basilar arteries detected by doppler ultrasonography were not included. Also patients with stroke, related to cardiac embolism were excluded from the study, since embolic infarcts results from completely different pathogenesis and these patients are generally younger.

As the serum MDA levels were measured 6 months after the acute cerebrovascular accident in our study, the increased level is probably independent of the central nervous system (CNS) lesions. The reason of the high MDA levels can be related with the risk factors of stroke and/or atherosclerosis itself. Prevalent risk factors of stroke contribute to oxidative cellular and tissue damage (Alexandrova and Bochev 2005). Though the relations among lipoperoxidation and DM, dyslipidemia, or HT are well known, it was also demonstrated that elevated levels of serum thiobarbituric acid-reactive substances in patients with stable coronary artery disease (CAD) were independent of traditional risk factors (Walter et al. 2004). Also, Bonithon-Kopp et al. (1997) demonstrated that in patients with carotid atherosclerosis without any history of coronary artery disease or stroke, lipoperoxidation products were significantly elevated. They concluded that their study provided some epidemiological data to support the hypothesis that lipid peroxidation was 
involved in early phases of atherosclerosis.

In this study, we also showed that there were no significant differences in serum MDA levels among the stroke patients with or without HT; with or without DM; with or without dyslipidemia, so we can assume that HT, DM or dyslipidemia solely cannot be responsible for the high serum MDA levels. However, it can not be concluded that these risk factors does not contribute to the elevated serum MDA levels. In each patient with cerebral atherosclerosis, the risk factor or factors affecting serum MDA levels can be different.

Similar serum MDA levels in both groups of chronic stroke patients (patients with lacunar or large infarct areas) reflect that lesions in CNS have no affect on the serum MDA levels in the chronic stage.

It is well known that, high plasma lipoperoxidation product levels predict the cardiovascular accident independently the known atherosclerotic risk factors (Mezzetti et al. 2001). High serum MDA levels were detected in the individuals whose parents had atherosclerosis (Davidenkova et al. 1990). So, high levels detected in chronic stroke patients might reflect a hereditary predisposition.

Elevated peroxidation products are related with red blood cell aggregation, and also activate NADPH-oxidase complex in phagocytes and initiate a series of accidents leading to the enhanced uptake of low density lipoprotein by macrophages leading to the formation of foam cells. By these mechanisms lipid peroxides contribute to the progression of atherosclerosis (Alexandrova and Bochev 2005).

In conclusion we demonstrated that serum MDA levels in chronic ischemic stroke patients with both lacunar and atherothrombotic large infarcts were elevated. To the best of our knowledge this is the first report showing that serum MDA levels of these two groups are not sigificantly different. Also serum MDA levels did not differ solely by the existence or nonexistence of HT, DM or hyperlipidemia.

\section{References}

Alexandrova, M.L., Bochev, P.G., Markova,V.I., Bechev, B.G., Popova, M.A., Danovska, M.P. \& Simeonova, V.K. (2003) Oxidative stress in the chronic phase after stroke. Redox Report., 8, 169-176.

Alexandrova, M.L. \& Bochev, P.G. (2005) Oxidative stress during the chronic phase of stroke. Free Rad. Biol. Med., 39, 297-316.

Belch, J., Mc Laren, M., Hanslip, J., Hill, A. \& Davidson, D. (1998) The white blood cell and plasma fibrinogen in thrombotic stroke: a significant correlation. Int. Angiol., 17, 120-124.

Bolokadze, N., Lobjanidze, I., Momtselidze, N., Solomonia, R., Shakarishvili, R. \& McHedlishvili, G. (2004) Blood rheological properties and lipidperoxidation in cerebral and systemic circulation of neurocritical patients. Clin. Hemorheol. Microcirc., 30, 99-105.

Bonithon-Kopp, C., Coudray, C., Berr, C., Touboul, P.J., Feve, J.M., Favier, A. \& Ducimetiere, P. (1997) Combined effects of lipid peroxidation and antioxidant status on carotid atherosclerosis in a population aged 59-71 y: The EVA Study. Etude sur le Vieillisement Arteriel. Am. J. Clin. Nutr., 65, 121-127.

Cherubini, A., Ruggiero, C., Polidori, C. \& Mecocci, P. (2005) Potential markers of oxidative stress in stroke. Free Rad. Biol. Med., 39, 841-852.

Davidenkova, E.F., Shafran, M.G. \& Veksler, B.M. (1990) Indicators of lipid peroxidation in the blood in hereditary predisposition to arteriosclerosis. Klin. Med. (Mosk)., 68, 34-38.

DeGraba, T.J., Fisher, M. \& Yatsu, F.M. (1992) Atherogenesis and strokes. In: Stroke Pathophysiology, Diagnosis and Management. 2nd ed., edited by H.J.M. Barnett, J.P. Mohr, B.M. Stein \& F.M. Yatsu. Churchill Livingstone, NY, pp. 29-48.

Demirkaya, S., Topçuoglu, M.A, Aydın, A., Ulas, U.H., Isimer, A.L. \& Vural, O. (2001) Malondialdehyde, glutathione peroxidase and superoxide dismutase in peripheral blood erytrocytes of patients with acute cerebral ischemia. Eur. J. Neurol., 8, 43-51.

Dotan, Y., Lichtenberg, D. \& Pinchuk, I. (2004) Lipid peroxidation cannot be used as a universal criterion of oxidative stress. Prog. Lipid Res., 43, 200-227.

Garcia, J.H., Ho, K.L. \& Caccamo, D.V. (1992) Pathology of stroke. In: Stroke Pathophysiology, Diagnosis and Management 2 nd ed., edited by H.J.M. Barnett, J.P. Mohr, B.M. Stein \& F.M. Yatsu. Churchill Livingstone, NY, pp. 125-145.

Iavorskaia, V.A., Belous, A.M. \& Mokhamed, A.N. (2000) The level of middle mass molecules and lipid peroxation in blood of patients with different forms of stroke. $\mathrm{Zh}$. Nevrol. Psikhiatr. Im. S. S. Korsakova, 100, 48-51.

Mezzetti, A., Zuliani, G., Romano, F., Costantini, F., Pierdomenico, S.D., Cuccurullo, F., Fellin, R. \& Associazione Medica Sabin (2001) Vitamin E and lipid peroxide plasma levels predict the risk of cardiovascular accidents in a group of healthy very old people. J. Am. Geriatr. Soc., 49, 533-537.

Mohr, J.P. (1992) Lacunes In: Stroke Pathophysiology, Diagnosis and Management 2nd ed., edited by H.J.M. Barnett, J.P. Mohr, B.M. Stein \& F.M. Yatsu. Churchill Livingstone, NY, pp. 539-560.

Mohr, J.P. \& Sacco, R.L. (1992) Classification of ischemic 
strokes. In: Stroke Pathophysiology, Diagnosis and Management 2nd ed., edited by H.J.M. Barnett, J.P. Mohr, B.M. Stein \& F.M. Yatsu. Churchill Livingstone, NY, pp. 271-283.

Ohkawa, H., Ohishi, N. \& Yagi, K. (1979) Assay for lipid peroxides in animal tissues by thiobarbituric acid reaction. Anal. Biochem., 95, 351-358.

Polidori, M.C., Cherubini, A., Stahl, W., Senin, U., Sies, H. \& Mecocci, P. (2002) Plasma carotenoid and malondialdehyde levels in ischemic stroke patients: relationship to early outcome. Free Radic. Res., 36, 265-268.

Re, G., Azzimondi, G., Lanzarini, C., Bassein, L., Vanoa, I. \& Guarnieri, C. (1997) Plasma lipoperoxidative markers in ischaemic stroke suggest brain embolism. Eur. J. Emerg. Med., 4, 5-9.

Sacco, R.L. (2000) Pathogenesis, classification, and epidemiology of cerebrovascular disease. In: Merrits's Neurology. 10th ed., edited by L.P. Rowland. Lippincot Williams \&
Wilkins, NY, pp. 217-229.

Sharpe, P.C., Mulholland, C. \& Trinick, T. (1994) Ascorbate and malondialdehyde in stroke patients. Ir. J. Med. Sci., 163, 488-491.

Tamam, Y., Iltumur, K. \& Apak, I. (2005) Assessment of acute phase proteins in acute ischemic stroke. Tohoku J. Exp. Med., 206, 91-98.

Walter, M.F., Jacob, R.F, Jeffers, B., Ghadanfar, M.M., Preston, G.M., Buch, J. \& Mason, R.P. (2004) Serum levels of thiobarbituric acid reactive substances predict cardiovascular accidents in patients with stable coronary artery disease: a longitudinal analysis of the PRACCIDENT study. J. Am. Coll. Cardiol., 44, 1996-2002.

Wehr, H., Ryglewicz, D., Rodo, M., Pozniak, M., Swiderska, M., Panczenko, B. \& Stajniak, A. (2000) In vitro oxidation of low density lipoproteins in patients after ischemic stroke. Neurol. Neurochir. Pol., 34, 447-456. 